\title{
InP-based Comb-locked Optical Super Channel Transmitter
}

\author{
Zhixin Liu $^{(1)}$, Selina G. Farwel( ${ }^{(2)}$, Michael J. Wale ${ }^{(2)}$, David J. Richardson ${ }^{(1)}$, and Radan Slavík ${ }^{(1)}$ \\ (1) Optoelectronics Research Centre, University of Southampton, Southampton, SO17 1BJ, U.K. \\ (2) Oclaro, Inc., Caswell, Towcester, NN12 8EQ, U.K. \\ Author e-mail address: $\underline{\text { z.liu } @ \text { soton.ac.uk }}$
}

\begin{abstract}
We demonstrate a comb-based transmitter with a potential to be integrated on a single InP photonic chip. Nyquist-shaped polarization-multiplexed 16QAM/64QAM signals are generated and transmitted over 300-km of SMF-28.

OCIS codes: (060.2330) Fiber optics communications; (130.6622) Subsystem integration and techniques; (140.3520) Lasers, injection-locked; (140.3600) Lasers, tunable
\end{abstract}

\section{Introduction}

Driven by the need to reduce the size and power consumption, optical transmission systems are increasingly exploiting integrated transmitter technologies over conventional lithium niobate $\left(\mathrm{LiNbO}_{3}\right)$ technology for transmitter subassembly [1]. In the last few years, various material platforms and transmitter structures have been proposed for coherent (amplitude and phase) modulation, including InP [2-3], GaAs [4], silicon [5], plasmonic [6], silicon organic hybrid [7], and injection-locked direct laser modulation [8]. Among these technologies, monolithically integrated transmitters in InP platform have shown outstanding maturity and reliability and are expected to be predominant in near future optical transmission systems [2-3,9].

An interesting option for future transmitter technology is the ability to mutually frequency-lock optical carriers, which can be achieved by employing frequency comb technology [10-11]. It has been shown that dense wavelength division multiplexing (DWDM) transmission systems with mutually frequency-locked optical carriers can potentially offer several advantages as compared to conventional laser-bank based DWDM systems, including higher spectral efficiency, bandwidth flexibility [12], and efficient compensation of fiber nonlinearity [11]. These performance aspects are highly significant for future high capacity, flexible grid optical networks. An integrated transmitter with frequency-locked optical carriers could provide an attractive approach in this context.

In this paper, we review the progress of our proposed integrated comb-based transmitter [13-14]. The key building blocks, i.e. the comb generator, tone demultiplexer and IQ modulators, were all demonstrated using discrete devices and PICs based on the InP platform, promising a potential to be fully integrated. The tone demultiplexing was realized by optical injection lock (OIL) a telecom-grade digital super-mode distributed Bragg reflector (DSDBR) tunable laser, which allows for flexible wavelength selection and potentially reduced laser phase noise. Dualpolarization Nyquist-shaped 16/64 QAM signals were generated and transmitted over 300-km SMF-28 [14].

\section{Transmitter Configuration}

Fig. 1a shows the schematics of our proposed comb-locked super channel transmitter. The transmitter has three function blocks: comb generator, tone demultiplexer, and IQ modulators. The comb generator comprises a tunable laser integrated with a dual-electrode Mach-Zehnder modulator (MZM) Broadband flat comb can be generated by driving individual arm using RF signals with appropriate power and phase difference [13]. In the tone demultiplexer, a multimode interferometer (MMI) splits and injects the comb to a set of tunable lasers. The wavelength of each laser is tuned to the wavelength of the selected comb tone so that the laser is injection-locked, giving an output which is frequency-locked to the selected tone (with comb spacings as small as $6.25 \mathrm{GHz}$ ). This process is relatively insensitive to the comb tone(s) power(s), thus strongly relaxing the requirements on the power per tone and the power uniformity. It also allows for a significant reduction in the linewidth of the locked laser(s) because of the inherently large OIL locking bandwidth [15]. Finally, the frequency-locked tunable lasers are launched into the respective InP IQ modulators for coherent modulation before combined by a multiplexer to form the super channel signals.

\section{Experimental setup}

The key building blocks for our transmitter are based on commercially available InP-based devices. We first experimentally studied the comb generation using a compact device that contains a telecom C-band tunable laser emitting $13 \mathrm{dBm}$ and a push-pull modulator with a $\mathrm{V}_{\pi}$ of $2.7 \mathrm{~V}$ and a bandwidth of $10 \mathrm{GHz}$ (shown as the comb generator in Fig.1) [13]. The modulator was driven by $10-\mathrm{GHz}$ RF signals with a power difference of $1 \mathrm{~dB}$. DC bias voltages and relative phase difference $\Delta \phi$ are optimized for generating a flat comb. The system experiment that 
focuses on tone demultiplexing and signal generation/transmission is shown in Fig.2. Although the transmission experiment was conducted using a $25-\mathrm{GHz}$ comb based on Fabry-Perot modulators [16], it does not affect the conclusion we draw in this paper, which focuses on the demonstration of the key building blocks. The generated comb tones had an identical linewidth of about $4 \mathrm{kHz}$ and a total output power of $11 \mathrm{dBm}$. Their power was attenuated before injection into the DS-DBR laser (Oclaro TL5000 iTLA packaged without any isolator) via an optical circulator. The optical circulator could be avoided by OIL via the rear laser facet as demonstrated in [17]. The modulator used was a dual-polarization InP IQ modulator with a $\mathrm{V}_{\pi}$ of $\sim 1.5 \mathrm{~V}$. The modulator was driven by an arbitrary waveform generator (AWG, Tektronix AWG7122C) that operated at 10-bit $12 \mathrm{GSa} / \mathrm{s}$, generating 10GBaud Nyquist-16QAM signals, yielding 1.2 samples per symbol. The transmission link comprised of four $75-\mathrm{km}$ spans of SMF-28 with the link loss compensated by three 16-dB gain in-line amplifiers (EDFAs). ASE noise was filtered with a $30-\mathrm{GHz}$ tunable optical band pass filter.

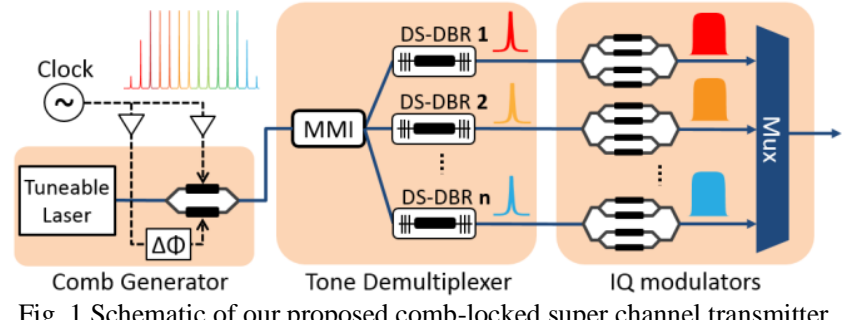

Fig. 1 Schematic of our proposed comb-locked super channel transmitter.

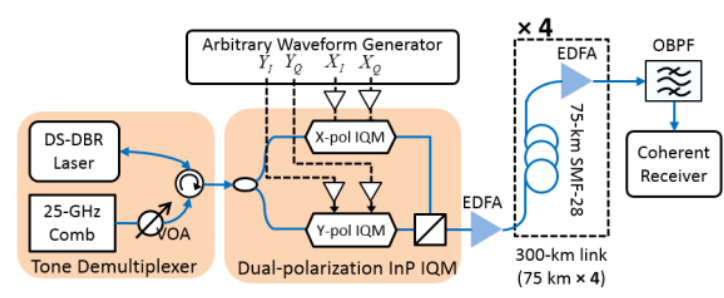

Fig.2 System experimental setup.

\section{Experimental results}

a) InP compact comb source

The comb generation using InP MZM involves a mixture of amplitude and phase modulation at each modulator electrode. In the experiment, we kept increasing the RF power for obtaining more comb tones up to the $26 / 27 \mathrm{dBm}$ level. Beyond this point no additional tones were generated and higher attenuation was observed. This is due to shift of the bandgap absorption wavelength when applying voltage on semiconductor material. For a given RF power, we could obtain an essentially flat comb spectrum as shown in Fig. 3 (bottom curve). By optimizing the DC bias voltages and the phase $\Delta \phi$. It was possible to get more comb tones by allowing a dip in the middle of the spectrum, Fig. 3, middle and upper curve. We also see that a wider comb with a more pronounced dip is generated with lower conversion efficiency. Using an off-the-shelf device, 29 tones (corresponds to $280 \mathrm{GHz}$ span) with less than $3 \mathrm{~dB}$ power ripple were obtained.

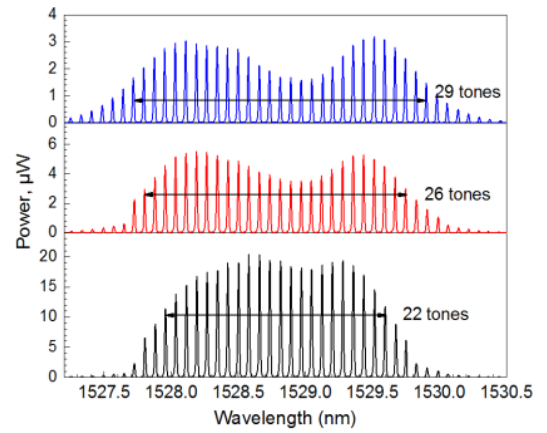

Fig. 3. Comb response when both RF signals were $26 / 27 \mathrm{dBm}$ for completely flat (22 tones) comb and comb with increased dip in the middle (26 and 29 tones)
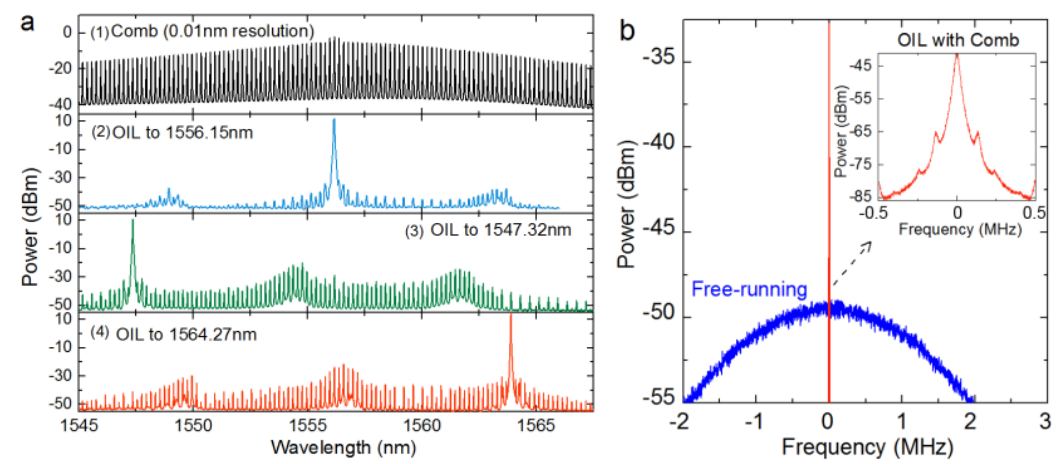

Fig. 4. (a) Optical spectra of the optical frequency comb and DS-DBR laser OIL-locked to three comb tones. (b) Measurement of the laser linewidth using the self-heterodyne method. blue: free-running, red: OIL with comb. The inset shows the result for the laser with OIL.

\section{(b) OIL-based tone extraction}

The optical spectrum (10 pm resolution) of the Fabry-Perot modulator based frequency comb (that we used to replace the compact comb due to device availability) is shown in subplot (1) in Fig. 4a. The center tone at 1556.15 $\mathrm{nm}$ had a power of $-2 \mathrm{dBm}$ and an OSNR $(0.1 \mathrm{~nm}$ resolution) of $24 \mathrm{~dB}$. The power and the OSNR of the comb tones decreased to $-14 \mathrm{dBm}$ and $15 \mathrm{~dB}$ at the $1547.32 \mathrm{~nm}$, and $-13 \mathrm{dBm}$ and $16 \mathrm{~dB}$ at $1564.27 \mathrm{~nm}$, respectively. The subplots (2)-(4) in Fig. 4a show the optical spectra of the comb and OIL-laser at a central wavelength of 1556.15 $\mathrm{nm}$, and at short/long wavelengths of $1547.32 \mathrm{~nm}$, and $1564.27 \mathrm{~nm}$, representing a tuning range of $17 \mathrm{~nm}$. Residual comb tones ( $>30 \mathrm{~dB}$ below the signal level) can be seen in the OIL laser spectrum - these are due to reflection from 
the front facet. It is believed this could be strongly suppressed if rear-facet OIL was employed [17]. The suppression ratios ('side-mode-suppression-ratio', SMSR) of the unwanted tones after OIL were between 30 and $48 \mathrm{~dB}$ (for OIL at 1547 and $1654 \mathrm{~nm}$, the strongest comb tones were at wavelengths close to the center of the comb at $1556 \mathrm{~nm}$ ). The linewidth of the free-running DS-DBR laser was measured to be $\sim 2.5 \mathrm{MHz}$ and this was significantly reduced to $4 \mathrm{kHz}$ (similar to the narrow linewidth of the seed laser) with OIL (shown in Fig. 4b). The single comb tone power injected into the DS-DBR was $-28 \mathrm{dBm}$, resulting in a (measured) locking range of $\sim 700 \mathrm{MHz}$.

\section{(c) Signal generation and transmission}

Fig. 5 shows the BER measurement results for the DP-Nyquist-16QAM. The closed markers show the measured results at back-to-back for 1556.15 (Fig. 5a), 1564.27 (Fig. 5b), and $1547.32 \mathrm{~nm}$ (Fig. 5c). The corresponding open markers show the results after transmission over 300-km of SMF-28. The launch power was optimized for the smallest BER (-4 dBm), at which an OSNR of $31 \mathrm{~dB}$ was obtained after transmission. At a BER of $3.8 \times 10^{-3}$, the required OSNR after the transmission was 23.6, 24.5, and $25.5 \mathrm{~dB}$ for $1556.15 \mathrm{~nm}, 1547.32 \mathrm{~nm}$, and $1564.27 \mathrm{~nm}$ respectively. The difference can be attributed to the EDFA gain profile. A preliminary result of DP-Nyquist-64QAM signals is shown in Fig. 6.
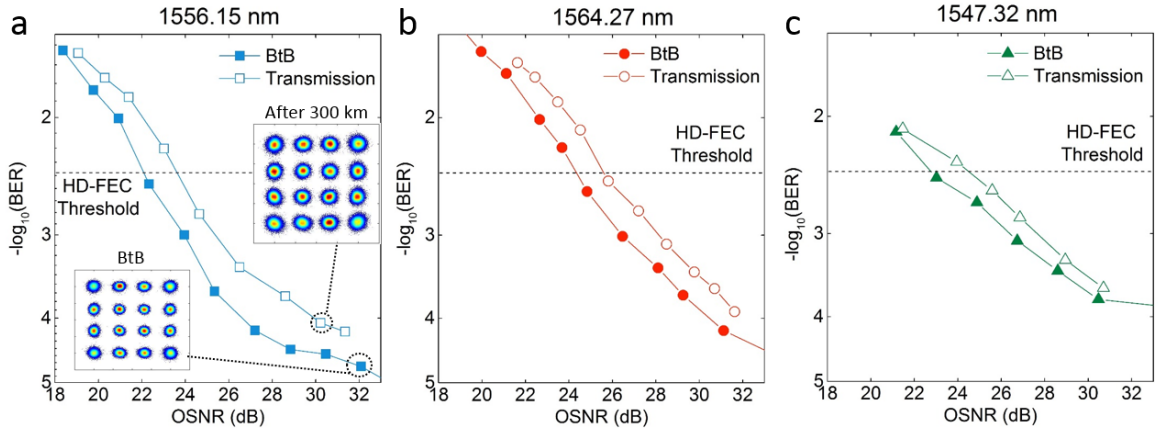

Fig. 5 Measured BER of the single-channel PDM-Nyquist-16QAM for three wavelengths for back-to-back (solid marker) and after transmission (open marker). (a) $1556.15 \mathrm{~nm}$; (b) $1564.27 \mathrm{~nm}$; (c) $1547.32 \mathrm{~nm}$.

\section{Conclusion}

The concept and key building blocks of a super-channel transmitter based on integrated comb and optical injection locking are demonstrated using InP devices. Sub-HD-FEC BER was achieved for PDM-Nyquist-16QAM signal transmission over 300-km of SMF-28. In the future, we plan on locking more lasers simultaneously to several comb tones; generating flexible DWDM and super-channel sources; and investigating the injection locking of DS-DBR lasers from the rear, in order to improve side-mode suppression and to eliminate the need for an optical circulator, thereby facilitating eventual monolithic integration using the InP platform.

\section{References}

[1] A. Chen and E. Murphy, Broadband Optical Modulators: Science, Technology, and Applications, Boca Raton, FL, USA: CRC Press, 2011.

[2] R. A. Griffin, et al., "InP Mach-Zehnder Modulator Platform for 10/40/100/200-Gb/s Operation," JSTQE, 19, 158-166 (2013).

[3] S. Chandrasekhar, et al., "Compact All-InP Laser-Vector-Modulator for Generation and Transmission of 100-Gb/s PDM-QPSK and 200-Gb/s PDM-16-QAM," JLT, 32, 736-742 (2014).

[4] P. C. Schindler, et al., "Monolithic GaAs Electro-Optic IQ Modulator Demonstrated at 150 Gbit/s With 64QAM," JLT, 32, 760-765 (2014).

[5] P. Dong, et al., "Silicon In-Phase/Quadrature Modulator With On-Chip Optical Equalizer," JLT, 33, 1191-1196 (2015).

[6] C. Haffner, et al., "All-plasmonic Mach-Zehnder modulator enabling optical high-speed communication at the microscale," Nature Photonics, 9, 525-528 (2015).

[7] S. Wolf, et al., "DAC-Less Amplifier-Less Generation and Transmission of QAM Signals Using Sub-Volt Silicon-Organic Hybrid Modulators," JLT, 33, 1425-1432 (2015).

[8] Z. Liu, et al., "Modulator-free quadrature amplitude modulation signal synthesis," Nature Communications, 5 (2014).

[9] S. G. Farwell, et al., "InP coherent receiver chip with high performance and manufacturability for CFP2 modules," OFC, W1I.6 (2014).

[10] J. Pfeifle, et al., "Coherent terabit communications with microresonator Kerr frequency combs," Nature Photonics, 8, 375-380 (2014).

[11] E. Temprana, et al., "Overcoming Kerr-induced capacity limit in optical fiber transmission," Science, 348, 1445-1448 (2015).

[12] N. Sambo, et al., "Sliceable Transponder Architecture Including Multiwavelength Source," JOCN, 6, 590-600 (2014).

[13] R. Slavík, et al., "Compact Optical Comb Generator Using InP Tunable Laser and Push-Pull Modulator," PTL, 27, 217-220 (2015).

[14] Z. Liu, et al., "InP-based Optical Comb-locked Tunable Transmitter," OFC, Tu2K.2 (2016).

[15] Z. Liu, et al., "Homodyne OFDM with Optical Injection Locking for Carrier Recovery," JLT, 33, 34-41 (2015).

[16] S. Xiao, et al., "Toward a low-jitter $10 \mathrm{GHz}$ pulsed source with an optical frequency comb generator," OE, 16, 8498-8508 (2008).

[17] A. Albores-Mejia, et al., "Optical-Comb-Line Selection from a Low-Power/Low-OSNR Comb using a Low-Coherence Semiconductor Laser for Flexible Ultra-Dense Short Range Transceivers," OFC, W2A.23 (2015). 